\title{
Can reduction in infection and mortality rates from coronavirus be explained by an obesity survival paradox? An analysis at the US statewide level
}

\author{
Yuval Arbel $\mathbb{D}^{1} \cdot$ Chaim Fialkoff $^{2} \cdot$ Amichai Kerner $^{3} \cdot$ Miryam Kerner $^{4}$
}

Received: 12 May 2020 / Revised: 20 July 2020 / Accepted: 3 September 2020 / Published online: 15 September 2020

(C) Springer Nature Limited 2020

Coronavirus disease 2019 (COVID-19) is a declared global pandemic with multiple risk factors. Based on recent empirical studies, obesity is considered by several researchers as one of the serious risk factors for coronavirus-related complications. Yet, other scholars argue in favor of the existence of an obesity survival paradox. The objective of the current study is to analyze the potential relationships between different corona indicators and obesity on a statewide level. Since the United States is ranked as one of the OECD countries with a high level of overweight and obesity among its citizens-the majority of US states exceed the $30 \%$ benchmark of obese population-it is an especially interesting case study to explore this issue. In an attempt to estimate projected probabilities for infection by coronavirus and mortality rates as a function of obesity prevalence, the fractional logit regression is employed. Findings may support the counter-intuitive possibility of an obesity survival paradox. Consequently, ethical guidelines referring to priority in intubation and intensive care treatments should account for these complex relationships between obesity and corona. Both projected rates of infection and mortality drop with elevated prevalence of obesity. The reasons for these findings might be explained by several conditions such as elevated social distancing from more obese persons, increased metabolic reserves, more aggressive treatment, and unidentified factors that should be examined in future research.

\section{Introduction}

Coronavirus disease 2019 (COVID-19) is a declared global pandemic with multiple risk factors (WHO report: coronavirus [1]). A summary of a report of more than 72,000 cases from the Chinese Center for Disease Control and Prevention reported by $\mathrm{Wu}$ and McGoogan [2] found that elderly persons ( $\geq 65$ years) and the presence of comorbidities of cardiovascular disease and diabetes mellitus are associated with a more severe course and higher fatality

Yuval Arbel

yuval.arbel@gmail.com

1 Sir Harry Solomon School of Economics and Management, Western Galilee College, Acre 2412101, Israel

2 Institute of Urban and Regional Studies, Hebrew University of Jerusalem, Mt. Scopus, Jerusalem 9190501, Israel

3 School of Real Estate, Netanya Academic College, 1 University Street, Netanya 4223587, Israel

4 The Ruth and Bruce Rappaport Faculty of Medicine, Technion-Israel Institute of Technology, Haifa, Israel rates of COVID-19 patients. As obesity is strongly associated with increased risk of cardiovascular disease and diabetes mellitus through direct effects (obesity induced structural and functional adaptions of cardiovascular system to accommodate excess body weight, adipokine effects on inflammation, and vascular homeostasis) and indirect effects (insulin resistance, hyperglycemia, hypertension, and dyslipidemia), a high body mass index (BMI), $\mathrm{BMI}=\frac{\mathrm{kg}}{\mathrm{meter}^{2}}$, might be an important risk factor for a severe course of COVID-19 disease. Yet, Stefan et al. [3] argue in favor of the counter-intuitive possibility of an obesity survival paradox: "Conversely, an obesity survival paradox has been observed in patients with pneumonia. That is, despite the increased risk of pneumonia and difficulties of intubation and mask ventilation, the risk of death in patients with obesity and pneumonia might be decreased".

The objective of the current study is to analyze the potential relationships between different corona indicators and obesity on a statewide level in the USA. The collected indicators are updated to May 5, 2020, and include: number of corona infections, the number of deaths from corona, and the population of each state. 
Because of the prevalence of obesity among its citizens, the United States provides an interesting case study. According to the WHO report: obesity and overweight [4], $\mathrm{BMI} \geq 25$, and $\mathrm{BMI} \geq 30, \frac{\mathrm{kg}}{\mathrm{meter}^{2}}$, are considered the respective benchmarks for overweight and obesity, based on which the prevalence of obesity is measured for each OECD country (a further discussion on prevalence of obesity is given in [5]). The USA is ranked as one of the most overweight and obese among OECD countries (0.226-0.381 prevalence of obesity) preceded only by Mexico and Chile. In fact, the prevalence of obesity in more than 30 US states exceeds $30 \%$, where one territory (Samoa) reaches to a maximum of $75 \%$.

The outcomes obtained from our study may support the counter-intuitive possibility of an obesity survival paradox. The reasons for the drop in infection and mortality rates with elevated prevalence of obesity might be explained by several conditions such as higher social distancing by more obese persons, increased metabolic reserves, more aggressive treatment, and unidentified factors that should be examined in future research.

The remainder of the article is organized as follows. "Methodology" section portrays the methodology. In "Results" section, results are given. The "Summary and conclusions" section gives conclusion and summary.

\section{Methodology}

Consider the following two competing fractional logit models (e.g., Papke and Wooldridge [6]; Johnston and
Dunardo (pp. 424-426) [7]; Wooldridge [8]):

$\operatorname{Pr}\left(0<Y_{j}<1\right)=F\left(\hat{\alpha}_{1 j}+\hat{\alpha}_{2 j} X\right)$,

$\operatorname{Pr}\left(0<Y_{j}<1\right)=F\left(\hat{\beta}_{1 j}+\hat{\beta}_{2 j} X+\hat{\beta}_{3 j} X^{2}\right)$,

where $\quad j=1, \quad 2 ; \quad Y_{1}=$ INFECTED $=\frac{\text { CORONA_CASES }}{\text { POPULATION }}$ $Y_{2}=$ MORTALITY $=\frac{\text { DEATH_CASES }}{\text { CORONA_CASES }} ; \quad X=$ prevalence of obese adults (2020) in each state $(\mathrm{BMI} \geq 30)$; $F\left(Y_{j}\right)=\frac{\exp \left(Y_{j}\right)}{1+\exp \left(Y_{j}\right)} ; \mathrm{OR}=\operatorname{proj}\left[\frac{p}{1-p}\right] ; \alpha_{1 j}, \alpha_{2 j}$, and $\beta_{1 j}, \beta_{2 j}$, and $\beta_{3 j}$ are parameters; and the circumflex denotes estimated parameters. Unlike Eq. (1), Eq. (2) permits nonmonotonic change of the corona indicator with the $X$ value.

\section{Results}

Based on the outcomes given in Table 1, Fig. 1 reports the projected probability to be infected from coronavirus vs. the prevalence of obesity in 2020 (BMI $\geq 30$ ) across the USA. The latter variable ranges between a minimum of 0.226 , indicating that the BMI $\geq 30$ for $22.6 \%$ of the population in Colorado, to a maximum of 0.381 , indicating that the $\mathrm{BMI} \geq 30$ for $38.1 \%$ of the population in West Virginia. Projections are based on the fractional logit regression of the linear model. As for the quadratic model, given that the calculated Wald Chi-square statistics (4.071) is lower than the critical $10 \%$ value with two degrees of freedom (4.6052)

Table 1 Regression analysis.

\begin{tabular}{lll}
\hline Variables & $(1)$ OR $\left(Y_{1}=\right.$ infected $)$ & (2) OR $\left(Y_{1}=\right.$ infected) \\
\hline Constant & $0.0271^{* * *}$ & 0.0473 \\
$X=$ Obese adults (2020) & $(0.00228)$ & $(0.591)$ \\
& $6.58 \times 10^{-4 * *}$ & $1.46 \times 10^{-5}$ \\
$X^{2}=$ [Obese adults (2020)] ${ }^{2}$ & $(0.0467)$ & $(0.773)$ \\
& - & 614.12 \\
Observations & - & $(0.921)$ \\
Calculated Wald Chi-square (d.f. $=1$ or 2) & 54 & 54 \\
Critical Chi-square (d.f. $=1$ or 2$)$ at the 10\% level & $3.955^{* *}$ & 4.071 \\
Critical Chi-square (d.f. $=1$ or 2) at the 5\% level & 3.84055 & 4.6052 \\
Critical Chi-square (d.f. $=1$ or 2) at the 1\% level & 6.6349 & 5.9915 \\
Descriptive statistics & & 9.2103 \\
Minimum prevalence of infection from coronavirus & 0.0004 & \\
Maximum prevalence of infection from coronavirus & 0.0164 & 0.0004 (Montana) \\
Minimum prevalence of obesity & 0.226 & 0.0164 (New York) \\
Maximum prevalence of obesity & 0.381 & 0.226 (Colorado) \\
\hline
\end{tabular}

Robust $p$ values are given in parentheses.

$* p<0.1 ; * * p<0.05 ; * * * p<0.01$. 
one cannot reject the null hypothesis that both included coefficients equal zero.

Results demonstrate that the projected probability to be infected from coronavirus drops with a higher prevalence of obesity, from 62 per 10,000 persons in states whose population exhibits $20 \%$ prevalence of obesity to only 17 per 10,000 persons in those states whose population has a $38 \%$ prevalence of obesity. Moreover, the null hypothesis of no drop with obesity prevalence is rejected statistically at the $5 \%$ level $(p=0.0467)$. Finally, note that the $95 \%$

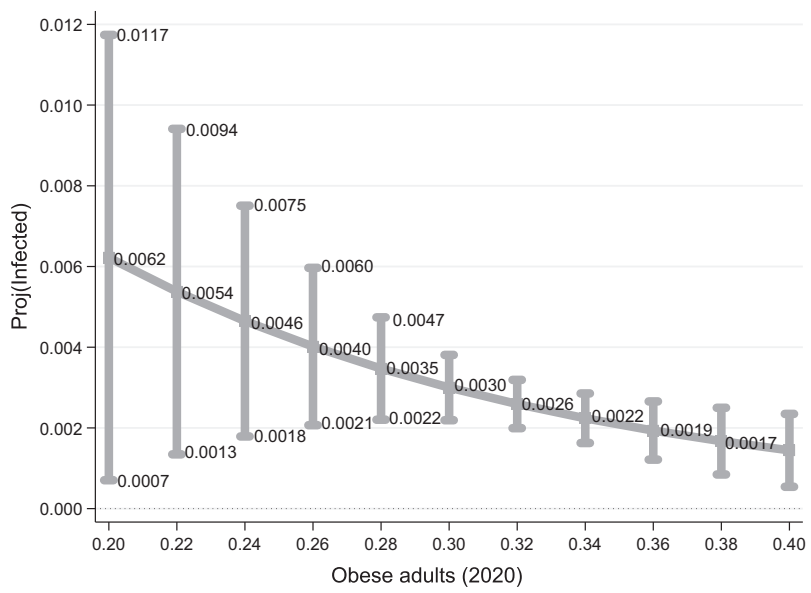

Fig. 1 Projected infections by coronavirus vs. prevalence of obese adults in US States. $Y_{1}=$ Infected $=\frac{\text { Corona Cases }}{\text { Population }}$ in each US state (excluding the outlier of Samoa). Projections are based on the following fractional logit regression analysis of the linear model given in column (1), where $\mathrm{OR}=$ odd ratio $=\operatorname{proj}\left[\frac{p}{1-p}\right]$. confidence interval spread around the projection is the highest for $20 \%$ prevalence of obesity (0.0007-0.0117) and drops considerably with an elevated prevalence of obesity. One possible interpretation of this finding is a smaller number of states with higher obesity prevalence. Consequently, we ran a symmetry distribution test for prevalence of obesity across the states. Note that the null hypothesis of symetrical distribution (calculated adjusted Chi-square statistic with two degrees of freedom is 1.21 and $p=0.5455$ ) is not rejected. The implication is that there are an equal and small number of states with low and high prevalence of obesity.

Based on the outcomes given in Table 2, Fig. 2 reports the projected probability of mortality rate from coronavirus vs. the prevalence of obesity in 2020 (BMI $\geq 30$ ) across US states. Projections are based on the fractional logit regression of the linear model. As for the quadratic model, given that both coefficients of $X$ and $X^{2}$ exhibit $p=0.226$ and $p=$ 0.292 respectively, one cannot reject the null hypothesis that both included coefficients equal zero.

Results demonstrate that projected probability of mortality from coronavirus drops with a higher prevalence of obesity: from 535 per 10,000 persons infected by coronavirus with a state population with a $20 \%$ prevalence of obesity to 346 per 10,000 persons in a state population with a $38 \%$ prevalence of obesity. Moreover, the null hypothesis of no drop with obesity prevalence is rejected statistically at the $10 \%$ level $(p=0.0733)$. Unfortunately, based on the $95 \%$ confidence intervals, the projected mortality rate from coronavirus at the statewide level is above $2 \%$ within all range of obesity prevalence.

Table 2 Regression analysis.

\begin{tabular}{lll}
\hline Variables & $(1) \mathrm{OR}\left(Y_{2}=\right.$ Mortality) & (2) OR( $Y_{2}=$ Mortality) \\
\hline Constant & $0.0938^{* * *}$ & 1.1307 \\
& $\left(1.39 \times 10^{-8}\right)$ & $(0.959)$ \\
$X=$ Obese adults (2020) & $0.0792^{*}$ & $4.08 \times 10^{-9}$ \\
& $(0.0733)$ & $(0.226)$ \\
$X^{2}=[\text { Obese adults }(2020)]^{2}$ & - & $1.22 \times 10^{-12}$ \\
& - & $(0.292)$ \\
Observations & 54 & 54 \\
Calculated Wald Chi-square (d.f. $=1$ or 2) & $3.207 *$ & $4.753 *$ \\
Critical Chi-square (d.f. $=1$ or 2) at the 10\% level & 2.7055 & 4.6052 \\
Critical Chi-square (d.f. $=1$ or 2) at the 5\% level & 3.8415 & 5.9915 \\
Critical Chi-square (d.f. $=1$ or 2) at the 1\% level & 6.6349 & 9.2103 \\
Descriptive statistics & & \\
Minimum mortality rate from coronavirus & 0.0079 & 0.0079 (South Dakota) \\
Maximum mortality rate from coronavirus & 0.0941 & 0.0941 (Michigan) \\
Minimum prevalence of obesity & 0.226 & 0.226 (Colorado) \\
Maximum prevalence of obesity & 0.381 & 0.381 (West Virginia)
\end{tabular}

Robust $p$ values are given in parentheses.

$* p<0.1 ; * * p<0.05 ; * * * p<0.01$. 


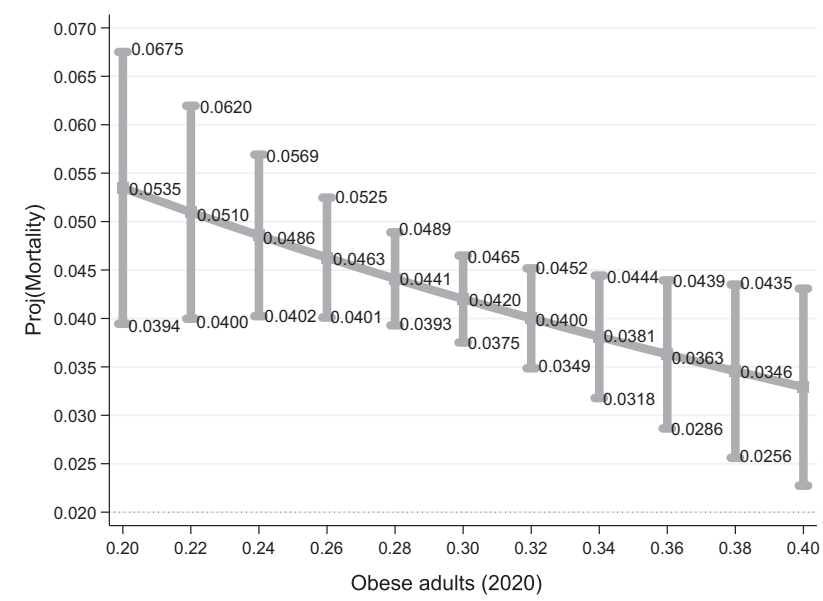

Fig. 2 Projected mortality rates vs. prevalence of obesity in US States. $Y_{2}=$ Mortality $=\frac{\text { Deaths from Corona in each US state (excluding the }}{\text { Cases of Corona }}$ outlier of Samoa). Projections are based on the following fractional logit regression analysis of the linear model given in column (1), where $\mathrm{OR}=$ odd ratio $=\operatorname{proj}\left[\frac{p}{1-p}\right]$.

\section{Summary and conclusions}

Following Simonnet et al. [9]; Garg et al. [10], and $\mathrm{Wu}$ and McGoogan [2], obesity may be considered as one of the serious risk factors causing coronavirus-related complications. Yet, Stefan et al. [3] points out that: "Conversely, an obesity survival paradox has been observed in patients with pneumonia. That is, despite the increased risk of pneumonia and difficulties of intubation and mask ventilation, the risk of death in patients with obesity and pneumonia might be decreased".

The objective of the current study is to examine the relationship between coronavirus indicators and the prevalence of obesity in the USA at a statewide level. Because of widespread obesity among its citizens, with more than 30 states having a BMI $\geq 30$ for at least $30 \%$ of the population, the USA is considered the third most overweight and obese state among OECD countries (0.226-0.381 prevalence of obesity, namely, BMI $\geq 30$, preceded only by Mexico and Chile). Consequently, it provides an interesting case study.

The outcomes obtained from our study may support the existence of an obesity survival paradox. The reasons for the drop in infection and mortality rates with an elevated prevalence of obesity might be explained by several conditions such as higher social distancing by more obese persons, increased metabolic reserves, more aggressive treatment, and unidentified factors that should be examined in future research.

Acknowledgements The authors are grateful to Yifat Arbel, Nissim Ben David (the president), and the participants at the "light at the corona tunnel" zoom meeting in Western Galilee College for helpful comments.

\section{Compliance with ethical standards}

Conflict of interest The authors declare that they have no conflict of interest.

Publisher's note Springer Nature remains neutral with regard to jurisdictional claims in published maps and institutional affiliations.

\section{References}

1. World Health Organization (WHO). Coronavirus. World Health Organization. 2020. https://www.who.int/health-topics/corona virus\#tab=tab_1.

2. Wu Z, McGoogan JM. Characteristics of and important lessons from the coronavirus disease 2019 (COVID-19) outbreak in china: summary of a report of 72,314 cases from the Chinese Center for Disease Control and Prevention. JAMA. 2020;323:1239-42. https://doi.org/10.1001/jama.2020.2648.

3. Stefan N, Birkenfeld AL, Schulze MB, Ludwig DS. Obesity and impaired metabolic health in patients with COVID-19. Nat Rev Endocrinol. 2020. https://doi.org/10.1038/s41574-0200364-6.

4. World Health Organization (WHO). Obesity and overweight. World Health Organization. 2020. https://www.who.int/newsroom/fact-sheets/detail/obesity-and-overweight.

5. Yuval A, Kerner A, Fialkoff C. The Association of Pension Income with the incidence of type I obesity among retired Israelis. J Obes. 2019:1-19. https://doi.org/10.1155/2019/5101867.

6. Papke LE, Wooldridge JM. Econometric methods for fractional response variables with an application to $401(\mathrm{k})$ plan participation rates. J Appl Econom. 1996;11:619-32.

7. Johnston J, Dunardo J. Econometric methods. 4th ed. Singapore: McGraw Hill; 1997.

8. Wooldridge JM. Econometric analysis of cross section and panel data. 2nd ed. Cambridge, MA: MIT Press; 2010.

9. Simonnet A, Chetboun M, Poissy J, Raverdy V, Noulette J, Duhamel A, et al. High prevalence of obesity in severe acute respiratory syndrome coronavirus-2 (SARS-CoV-2) requiring invasive mechanical ventilation. Obesity. 2020. https://doi.org/10. 1002/oby.22831.

10. Garg S, Kim L, Whitaker M, O'Halloran A, Cummings C, Holstein $\mathrm{R}$, et al. Hospitalization rates and characteristics of patients hospitalized with laboratory-confirmed coronavirus disease 2019 -COVID-NET, 14 States, March 1-30, 2020. Morb Mortal Wkly Rep. 2020;69:458-64. https://www.cdc.gov/mmwr/volumes/69/w $\mathrm{r} / \mathrm{mm} 6915 \mathrm{e} 3 . \mathrm{htm}$ ?s_cid=mm6915e3_w. 